\title{
Les ordres mendiants en Hongrie et la littérature médiévale en langue vernaculaire $\mathrm{XIII}^{\mathrm{e}}-\mathrm{XV}^{\mathrm{e}}$ siècle) ${ }^{1}$
}

\author{
Edit MADAS
}

Sur les quatre textes les plus anciens qui nous sont restés en langue hongroise, trois sont liés aux ordres mendiants. Le premier d'entre eux - un sermon funèbre composé vers $1200^{2}$ - a précédé l'installation en Hongrie des ordres dominicain et franciscain qui eut lieu dans les années 1220. À l'époque, l'usage de l'écriture en Hongrie se faisait presque uniquement en latin, ceux qui savaient lire et écrire savaient le latin et étaient à coup sûr des clercs. Les textes en langue hongroise qui avaient été inscrits opportunément dans un codex latin relevaient de la culture ecclésiastique. Ces textes virent le jour aux points de contact de l'écriture latine et de l'oral hongrois, et le plus souvent leur transcription était liée à une activité de prédication. De fait, la prédication est un genre qui repose sur des sermons écrits en latin, mais qui à l'oral s'adresse aux fidèles ne parlant pas latin dans leur langue maternelle. Le premier poème en langue hongroise, le Ómagyar Mária-siralom [Complainte de Marie en ancien hongrois], a été transcrit par un frère dominicain à la fin du premier tome d'un sermonnaire en latin en deux volumes du XIII ${ }^{\mathrm{e}}$ siècle $^{3}$.

Ce petit volume a longtemps été sans être relié, de sorte que le texte hongrois figurant sur la dernière page a été partiellement effacé par le frottement des mains des usagers. Le sermonnaire contient des sermons de théologiens dominicains français et italiens du XIII ${ }^{e}$ siècle, tels Aldobrandinus de Cavalcantibus, Constantinus de Orvieto, Hugues de Saint-Cher, Johannes de Opreno, etc. D'après l'écriture, la sélection, vraisemblablement italienne, pourrait dater du troisième quart du XIII ${ }^{\mathrm{e}}$ siècle. Rapidement les deux volumes arrivèrent en Hongrie où ils furent, dans le dernier quart du XIII ${ }^{\mathrm{e}}$ siècle, complétés de quelque 50 sermons latins par deux dominicains hongrois, dont l'un nota aussi le poème en langue hongroise ${ }^{4}$. À côté des sermons on trouve des gloses en hongrois, et c'est en raison de ses tournures faciles à exploiter au cours de la prédication que le poème a pu trouver place dans le manuscrit. Paraphrase de la séquence Planctus sancte Marie [Planctus ante nescia d'après l'incipit] de Godefroid de Saint-Victor, mort vers 1194, la Complainte de Marie est une transposition libre, l'œuvre

\footnotetext{
1. Cette contribution a été préparée dans le cadre des activités du groupe de recherche « Res libraria Hungariae » (Académie des Sciences de Hongrie - Bibliothèque nationale de Hongrie).

2. « Látjátok feleim... » Magyar nyelvemlékek a kezdetektől a 16. század elejéig ["Vous le voyez, mes frères... », monuments de la langue hongroise des origines au début du XVI ${ }^{\mathrm{e}}$ siècle]. Kiállítási katalógus, az Országos Széchényi Könyvtár kiállítása, 29 octobre 2009-28 février 2010, Edit Madas éd., Budapest, 2009, nº 5-6.

3. « Látjátok feleim... », $\mathrm{n}^{\circ} 7-8$.

4. Les deux volumes furent reliés en un seul au cours du XIV siècle. Le Leuveni Kódex (Codex de Louvain) tire son nom du lieu où il fut conservé de 1922 à 1982 : Budapest, Bibliothèque nationale de Hongrie, MNY 79. András Vizkelety, Az európai prédikációirodalom recepciója a Leuveni Kódexben - Die Rezeption der Europäischen Sermonesliteratur im «Löwener Kodex », Budapest, 2004 (Fragmenta et codices in bibliothecis Hungariae, 4).
} 
d'un poète à l'aise dans la versification hongroise et maîtrisant bien la poésie latine. À en juger par la langue, le poème avait déjà au moins 50 ans au moment où il fut mis par écrit, il avait donc d'abord connu une longue vie dans sa version orale. « f »

L'ensemble des textes suivants, environ 30 ans plus tard, se rattache plus étroitement encore à la prédication : il s'agit de trois distinctiones rimées sur les marges d'un sermonnaire franciscain ${ }^{5}$. Les Gyulafehérvári Sorok [vers de Gyulafehérvár] doivent leur nom au lieu où ils sont conservés : Gyulafehérvár, aujourd'hui Alba Iulia en Roumanie. Parmi les auteurs des sermons, quatre sont maîtres régents à la faculté de théologie de l'université de Paris : saint Bonaventure, Jean de la Rochelle, Eudes Rigaud et Gilbert de Tournai ${ }^{6}$. Le sermonnaire fraîchement composé de leurs sermons a pu être emporté en Hongrie par un franciscain hongrois étudiant à Paris, et vers 1320 on réalisa un nouveau codex sur la base du manuscrit parisien dans lequel furent copiées, à côté des sermons, des formules de charte propres à la Hongrie. Dans la marge de trois sermons différents se trouvent trois sortes de distinctiones rimées. La première est pour la Circoncision, qui est aussi la fête de l'« impositio nominis Jesu ». Dans la marge le prédicateur a copié en latin, puis traduit en hongrois, un acrostiche sur le nom de Jésus. Dans la marge inférieure du sermon écrit pour la fête de saint Thomas, le frère franciscain traduit en hongrois la division rimée en latin qui se trouve dans le sermon. La question est de savoir comment l'on peut croire aujourd'hui en la Résurrection du Christ si on doute, à l'instar de l'apôtre Thomas. La réponse se présente sous forme d'un quatrain :

\footnotetext{
In mirifici operis indicio

In verbi oris sui testimonio

In sacri altaris mysterio

In sui imaginis vestigio.
}

La traduction hongroise dénote que la technique du sermon est la même dans les deux cas et que le prédicateur, à cette occasion, change simplement de langue. La troisième distinctio hongroise, dans la marge d'un sermon sur la passion, n'a pas d'antécédent latin direct, elle est d'emblée l'œuvre du franciscain hongrois lui-même.

Naturellement, durant tout le Moyen Âge, les utilisateurs de sermonnaires maîtrisant bien le latin écrivirent régulièrement dans leurs volumes en latin des gloses, des textes en hongrois plus ou moins longs, en général à leur propre usage. Toutefois, à partir du début $\mathrm{du} \mathrm{XV}^{\mathrm{e}}$ siècle, nous sommes témoins d'un phénomène nouveau : le besoin d'une nourriture spirituelle dans des cercles qui n'avaient pas toujours à leur disposition un clerc parlant latin. La lecture à haute voix en langue maternelle pouvait remplacer le recours à un prédicateur ou à un traducteur occasionnel. Le premier livre en langue hongroise parle de la vie de saint François et de ses compagnons, la traduction a été réalisée vers 1380, l'exemplaire qui nous est resté est une copie datant de 1440 environ? ${ }^{7}$ Les sources en sont les Actus

5. Alba Iulia, Bathyaneum, R. III. 89. Robertus Szentiványi, Catalogus concinnus librorum manuscriptorum Bibliothecae Batthyányanae, Szeged, 1958, n 395 ; « Látjátok feleim... », nº 9.

6. Edit Madas, «A XIII-XIV. századi magyarországi ferences prédikáció forrásvidéke » [Le domaine des sources de la littérature sermonnaire franciscaine hongroise des XIII -XIV siècles], Irodalomtörténeti Közlemények 97 (1993), p. 1-15.

7. Jókai-kódex [Codex Jókai] : Budapest, Bibliothèque nationale de Hongrie, MNY 67. Látjátok feleim..., nº 20 ; Jókaikódex (fac-similé), Dénes Szabó éd., Budapest, 1942 (Codices Hungarici, 1) ; Jókai-kódex XIV-XV. század (fac-similé, transcription et source latine), János P. Balázs éd., Budapest, 1981 (Codices Hungarici, 8) ; László Szörényi, « La problematica 
beati Francisci et sociorum eius, le Speculum perfectionis, la Légende de saint François de Bonaventure, ainsi que le Liber conformitatum de Barthélemy de Pise. La source latine directe nous est inconnue. Outre le contenu, de nombreux autres indices nous révèlent que la traduction et la copie sont l'une et l'autre le travail d'un franciscain.

La traduction est particulièrement lourde, pleine de latinismes et même de véritables contresens. Le traducteur n'avait pas une bonne maîtrise du latin, mais la situation avait aussi complètement changé par rapport aux œuvres précédentes. Il ne s'agit pas ici de l'adaptation ou de l'interprétation libre d'un texte, mais d'une traduction fidèle, mot à mot, phrase après phrase. Il n'était déjà plus question d'envie ni d'inspiration, mais d'une obligation, qui donna bien de la peine au traducteur. Cette traduction, unique à son époque, précède de loin la littérature conventuelle en langue hongroise qui s'épanouit dans le dernier tiers du Xve et le premier tiers du XvI ${ }^{\mathrm{e}}$ siècle. Elle conserve un état de langue beaucoup plus ancien, ce qui lui donne une place particulièrement importante dans l'histoire de l'écriture en langue hongroise. Mais pour la même raison il est difficile de déterminer pour qui véritablement la traduction a été réalisée à l'intérieur de l'ordre franciscain. Est-ce le fruit du hasard si le premier livre en langue hongroise est précisément ce codex franciscain ? Si nous considérons sa destinée, oui. En 1851, alors que des écoliers faisaient une bataille pendant la récréation, l'un d'eux lança précisément ce codex à son camarade ; ce dernier le rapporta chez lui et il apparut bientôt quel trésor il avait sauvé de la destruction. Cet événement représente d'ailleurs l'âge héroïque de la découverte des codices en langue hongroise. Auparavant personne ne s'y intéressait.

Si nous sommes dans l'incertitude en ce qui concerne les circonstances de la traduction de la Légende de saint François, on peut clairement voir, avec l'augmentation du nombre de sources à partir de la fin $\mathrm{du} \mathrm{Xv}^{\mathrm{e}}$ siècle, le rôle déterminant des ordres mendiants dans la création d'une littérature conventuelle en langue hongroise. En tout, ce sont près de 50 manuscrits en langue hongroise qui nous sont restés de la période qui s'étend de 1440 à 1530 . Le contenu des codices est religieux. Dans leur ensemble, les textes sont presque tous des traductions et presque tous aussi des copies. Cela suppose, d'une part, un milieu de lecteurs relativement restreint et homogène, bien qu'originellement il y ait eu beaucoup plus de volumes que ce que nous connaissons aujourd'hui. D'autre part, nous constatons que les traducteurs ont acquis en l'espace de quelques décennies une routine extraordinaire : pratiquement, c'est grâce à eux que la langue hongroise écrite a vu le jour. Les trois quarts des manuscrits qui nous sont restés peuvent être rattachés aux ordres mendiants, et les réformes observantes de ces ordres jouèrent assurément dans leur production un rôle important.

La plupart des manuscrits conservés ont été écrits à l'intention des sœurs dominicaines de l'île aux Lièvres et des clarisses de Óbuda, presque dans le même temps ; les deux couvents s'empruntaient même des livres. Nous parlerons avant tout des sœurs dominicaines, dont nous savons plus de choses, et nous ne mentionnerons les manuscrits des clarisses qu'à des fins de comparaison.

Les ruines des deux couvents sont aussi présentes dans la capitale du pays, à Budapest : le couvent dominicain sur l'île Sainte-Marguerite, à l'époque l'île aux Lièvres ; le couvent des clarisses juste en face, sur la rive opposée du Danube, à Óbuda ${ }^{8}$. Le couvent dominicain fut fondé par Béla IV, sous le patronage de la Vierge Marie, pour sa fille Marguerite devenue plus tard sainte Marguerite. En 1241, à l'époque des dévastations tartares, le couple royal avait fait le vœu, s'il survivait aux événements guer-

del codice "Jókai" alla luce degli studi recenti sulle leggende di San Francesco ", dans Spiritualità e lettere nella cultura Italiana e Ungherese del basso medioevo, Sante Graciotti et Cesare Vasoli éd., Firenze, 1995, p. 133-147.

8. En 1873, Pest, situé sur la rive est du Danube, fut réuni à Buda et à Óbuda situées sur la rive ouest. 
riers, de consacrer à Dieu leur fille à naître. En 1252, Marguerite s'installa dans le nouveau couvent annexé à une demeure royale et une maison conventuelle pour quelques frères. En 1270, quatre-vingt villages dépendaient du couvent, dont l'importance grandit encore après la mort de Marguerite grâce aux miracles qui se produisaient près de sa tombe. Malgré les tentatives infructueuses de canonisation - elles n'aboutirent qu'en 1943 - on l'honorait comme une sainte et sa tombe était devenue un lieu de pélerinage. Le couvent conserva un rôle marquant durant tout le Moyen Âge. On y éleva en grand nombre princesses et nobles demoiselles d'illustre naissance venant des quatre coins du pays ; une partie d'entre elles furent ensuite mariées.

Le couvent des clarisses de Óbuda fut fondé en 1334 par la mère du roi Louis le Grand (1342-1382), la reine Élisabeth. Veuve en 1342, la reine établit sa cour de veuve à Óbuda, et elle munit le bâtiment terminé en 1346 de merveilleux trésors, dont l'autel domestique décoré de statues enchâssées qui est aujourd'hui conservé au Metropolitan Museum de New York. La reine se fit enterrer dans ce couvent qui, grâce à ses donations, devint le plus prestigieux de son ordre.

Les frères de la province dominicaine hongroise, dans leur couvent de Buda - dont relevait aussi le contrôle de l'île aux Lièvres - mirent à exécution en 1454, sous la direction des dominicains de Vienne, la réforme qui visait à une stricte observance de la règle et des constitutions. La branche féminine s'efforça de se soustraire aux tentatives de réforme, et les sœurs reçurent l'autorisation du pape Pie II de choisir elles-mêmes leur prieure et de prendre un confesseur chez les franciscains. En 1468, le chapitre général autorisa le prieur provincial hongrois à exclure de l'ordre les sœurs du couvent de l'île aux Lièvres si elles ne se pliaient pas à la règle.

Le texte le plus important de l'activité réformatrice précédant l'épanouissement de la littérature conventuelle est un fragment de quatre feuillets appelé codex Birk ${ }^{9}$. En fait, ce texte marque la renaissance de l'île aux Lièvres. Les feuilles de papier noircies d'une écriture cursive contiennent de manière fragmentaire la traduction de la règle de saint Augustin et des constitutions de l'ordre dominicain. Cette traduction a été réalisée pour que les sœurs maîtrisant mal le latin comprennent et puissent suivre les prescriptions. La lecture de la règle de saint Augustin était faite une fois par semaine en communauté ; la lecture des constitutions, répartie sur un mois. La traduction a été réalisée en 1474 par le frère dominicain Pál de Vác, sur l'île aux Lièvres, dans la petite maison des frères qui se trouvait à côté du couvent des sœurs dominicaines.

Le manuscrit conservé est le premier brouillon de cette traduction. Au vu des suppressions et corrections qui y ont été faites, il apparaît clairement que le travail n'est pas encore terminé. Une version mise au propre de ce brouillon existait à Presbourg, comme en témoigne l'historiographe de l'ordre dominicain, Sigismond de Ferrare, qui rapporte, en 1636, y avoir vu la traduction mot à mot en langue hongroise de la règle d'Augustin et des constitutions ${ }^{10}$. À cette époque, les manuscrits de l'île SainteMarguerite se trouvaient en effet à Presbourg. Mais ce codex est aujourd'hui perdu. Le codex Birk apparaît à Vienne en 1889 dans la bibliothèque impériale, parmi divers défaits de reliure collectés à partir de 1860 à l'initiative d'Ernst Birk, alors directeur de la bibliothèque. Le frère dominicain Pál de Vác est le seul traducteur dont nous connaissions non seulement le nom mais aussi la vie d'une manière relativement détaillée. En 1448, il s'inscrivit à l'université de Vienne et la même année entra dans l'ordre dominicain. En 1450, il fut envoyé à l'université de Heidelberg où, deux ans plus tard, il

9. Birk-kódex : Budapest, Bibliothèque nationale de Hongrie, MNY 71. Látjátok feleim..., nº 29 ; Birk-kódex (fac-similé, transcription et source latine), István Pusztai éd., Budapest, 1960 (Codices Hungarici, 5).

10. Sigismundus Ferrarius, De Rebus Ungaricae Provinciae Sacri Ordinis Praedicatorum, Viennae, 1637, p. 449, 529. 
fut reçu magister artium. Vers 1474, il était sur l'île aux Lièvres où il s'occupait de soigner les âmes des sœurs dominicaines. En 1478, il reçut du pape Sixte IV le titre de magister theologiae et devint prieur du couvent de Nagyszombat. En 1480, il enlumina un exemplaire de la Somme de théologie de Thomas d'Aquin, édité à Venise en 1477. En 1482, en sa qualité de gardien des réformes, il dut prendre part, sur l'ordre de ses supérieurs, à la visite canonique des couvents dominicains de Buda et de Pest.

Pál de Vác mérite qu'on se soit un peu attardé sur son cas : ce dominicain universitaire, personnage-clé de la réforme observante, n'utilisait plus de manuscrits à la fin $\mathrm{du} \mathrm{Xv}^{\mathrm{e}}$ siècle, mais des livres imprimés, et qui plus est, il pouvait se les procurer peu de temps après leur parution. En revanche, le nombre des codices traduits pour les sœurs étaient si faible que la question de leur impression ne se posait même pas.

L'essentiel de la réforme de l'observance consistait partout dans le retour à la discipline primitive : réciter régulièrement l'office, renoncer aux biens personnels et observer strictement la clôture. Pour encourager la vie religieuse volontaire on prescrivait d'écouter des lectures édifiantes pendant le repas et dans la salle capitulaire. Toutefois, écouter la règle et les lectures favorisant l'ascèse et le renoncement au monde ne pouvait être efficace que si les sœurs comprenaient ce qu'on leur lisait, autrement dit, si on leur parlait dans leur langue maternelle. La traduction de la règle était disponible dès 1474 . Pour les trente années qui suivirent, il ne nous reste aucun livre provenant de l'île Sainte-Marguerite. En revanche nous connaissons quinze manuscrits réalisés entre 1506 et 1531 : douze servaient à la lecture en communauté, les trois autres étaient des livres de prières pour un usage personnel. Les textes furent traduits par des frères, en général à partir de livres imprimés, et à l'exception de trois manuscrits ils furent copiés par des sœurs. On distingue dans ce corpus les mains de vingt neuf sœurs. Parmi les textes destinés à la lecture, deux vies de saints se distinguent : celle de la sainte du lieu, Marguerite de Hongrie, datée de $1510^{11}$, et celle de saint Dominique, écrite en $1517^{12}$. Les sources principales de la Vie de sainte Marguerite en langue hongroise sont la Legenda vetus que le confesseur de Marguerite, le provincial Marcellus, écrivit tout de suite après sa mort (1270) pour la faire canoniser, et le procès-verbal d'audition de témoins de l'année $1270^{13}$. La légende du fondateur de l'ordre, elle, remonte à plusieurs sources, dont la plus importante est le Chronicon de l'archevêque Antonin de Florence († 1459). Dans le codex d'Érsekújvár ${ }^{14}$, la Légende de sainte Catherine de Sienne repose sur la même source, mais le traducteur-compilateur a aussi puisé dans le Dialogue de cette même sainte Catherine. Des lectures du couvent, il reste, dans le Codex Virginia ${ }^{15}$, une volumineuse Légende de saint François

11. Szent Margit élete [Vie de sainte Marguerite], Budapest, Bibliothèque nationale de Hongrie, MNY 3. Látjátokfeleim..., $\mathrm{n}^{\circ} 31$; Szent Margit élete 1510 (fac-similé et transcription), János Balázs, Adrienne Dömötör et Katalin Pólya éd., Budapest, 1990 (Régi Magyar Kódexek, 10).

12. Szent Domonkos élete [Vie de saint Dominique], Budapest, Bibliothèque nationale de Hongrie, MNY 6. Látjátok feleim..., n 31 ; Szent Domonkos élete (fac-similé et transcription), Gyöngyi Komlóssy éd., Budapest, 1990 (Régi Magyar Kódexek, 9).

13. Gábor Klaniczay, « Le dossier de la princesse Marguerite († 1270) », dans G. Klaniczay-E. Madas, La Hongrie, dans Hagiographies. Histoire internationale de la littérature hagiographique latine et vernaculaire en Occident des origines à 1550, Guy Philippart éd., t. 2, Turnhout, 1996, p. 123-130.

14. Érskújvári [aujourdhui Nové Zámky] Kódex, Budapest, Bibliothèque de l'Académie des Sciences de Hongrie, K 45. Látjátok feleim..., n 35 ; Érskújvári Kódex 1529-1531 (fac-similé et transcription), à paraître (Régi Magyar Kódexek, 30).

15. Budapest, Bibliothèque de l'Académie des Sciences de Hongrie, K 40. Virginia-kódex XVI. század eleje (fac-similé et transcription), Zsuzsa Kovács éd., Budapest, 1990 (Régi Magyar Kódexek, 11). 
copiée à partir d'un exemplaire emprunté au couvent des clarisses de Obuda. L'œuvre n'est pas identique à la Légende de François mentionnée plus haut.

Le couvent n'avait pas - semble-t-il - de recueil de légendes raisonné, qui embrassât toute l'année. Le codex Cornides (Préciser la cote du ms.) et le codex d'Érsekújvár contiennent des légendes principalement recueillies pour des fêtes de saintes, avec des sources variées allant de la Légende dorée à Pelbárt de Temesvár (Une référence bibliographique pour ce personnage ?) La Légende de sainte Catherine d'Alexandrie (codex d'Érsekújvár), avec plus de 4074 vers, se distingue des autres légendes ${ }^{16}$. Il en existe de nombreux parallèles étrangers, mais elle est unique en son genre dans l'histoire littéraire hongroise. Le second groupe de lectures concernait les fêtes de l'année ecclésiastique. Les plus importantes d'entre elles sont les méditations de la passion du Christ, les évangiles apocryphes (Actus Pilati, Descensus Christi ad inferos), les complaintes de Marie. Une partie de ces lectures est en relation directe avec les codices des clarisses de Óbuda. En général, les traductions des sermons pour Noël, Pâques, l'Ascension, la Pentecôte et d'autres fêtes servaient de lecture en plus des traductions de l'épître, de l'évangile et de la séquence de la fête. Leurs sources sont les sermonnaires du dominicain Vincent Ferrier?, de Jean Herolt et du grand prédicateur franciscain hongrois de l'époque, Pelbárt de Temesvár († 1504). L'ouvrage de Pelbárt consacré à la Vierge Marie, Stellarium Beatae Mariae Virginis, est la source des traités qu'on peut lire dans le codex Horvát ${ }^{17}$ sur l'incarnation du Christ et la mort de Marie. Nous avons conservé un nombre bien plus grand de méditations de Pelbárt sur Marie dans les manuscrits des clarisses.

Les écrits qui complètent la règle et éduquent à la vie religieuse relèvent des lectures indépendantes des fêtes : ce sont par exemple la Regula monacharum attribuée à saint Jérôme (codex Virginia), la Formula novitiorum de David d'Augsbourg (codex Horvát) ou le De modo bene vivendi du pseudosaint Bernard (codex d'Érsekújvár). Parallèlement aux écrits éduquant à la bonne vie, les écrits préparant à la bonne mort - prières du mourant et ars moriendi - reçoivent aussi une place importante.

Deux autres ouvrages intéressants méritent encore d'être signalés. Le premier est une version du Dultitius de Hrotsvita, chanoinesse de Gandersheim au $x^{\mathrm{e}}$ siècle, modernisée par l'humaniste Conrad Celtis. Comme Conrad Celtis se trouvait à Buda en 1497, il est possible qu'il ait lui-même apporté le texte. L'auteur de la variante hongroise l'a adapté aux conditions de son époque, le théâtre de l'œuvre étant non plus le palais impérial romain mais la cour du sultan $\operatorname{turc}^{18}$. Le second, bien construit et spirituel, est le traité sur la Dignité des saints apôtres, qui a peut-être été écrit directement à l'intention des sœurs de l'île aux Lièvres ${ }^{19}$.

Un scriptorium a fonctionné dans le couvent de l'île pendant plusieurs générations. La sœur copiste la plus remarquable, Lea Ráskay, bibliothécaire du couvent, était issue d'une famille de la haute

16. Régi Magyar Költök Tára [Trésor des anciens poètes hongrois], Cyrill Horváth éd., Budapest, 1921, p. 246-416.

17. Horvát-kódex, Budapest, Bibliothèque nationale de Hongrie, MNY 7. Horvát-kódex 1522 (fac-similé et transcription), Lea Haader et Zsuzsa Papp éd., Budapest, 1994 (Régi Magyar Kódexek, 17).

18. Sándor-kódex [Codex Sándor], Budapest, Bibliothèque universitaire Eötvös Loránt, Cod. Hung. 6. Látjátok feleim..., n 44 ; Sándor-kódex XVI. század első negyede (fac-similé et transcription), István Pusztai éd., Budapest, 1987 (Régi Magyar Kódexek, 3).

19. Könyvecse, Budapest, Bibliothèque universitaire Eötvös Loránt, Cod. Hung. 5. Látjátok feleim..., $\mathrm{n}^{\circ} 51$; Könyvecse az szent apostoloknak méltóságokról 1521 (fac-similé, transcription et source latine), István Pusztai éd., Budapest, 1985 (Régi Magyar Kódexek, 1). 
noblesse ${ }^{20}$. C'est elle qui apposa dans l'édition de la règle parue à Milan en 1505 par les soins de Vincentius Bandellus la note de possesseur du couvent. Il en reste cinq exemplaires qui forment la base de la bibliothèque. En général, ce sont des œuvres homogènes dont le contenu est stable. Lea

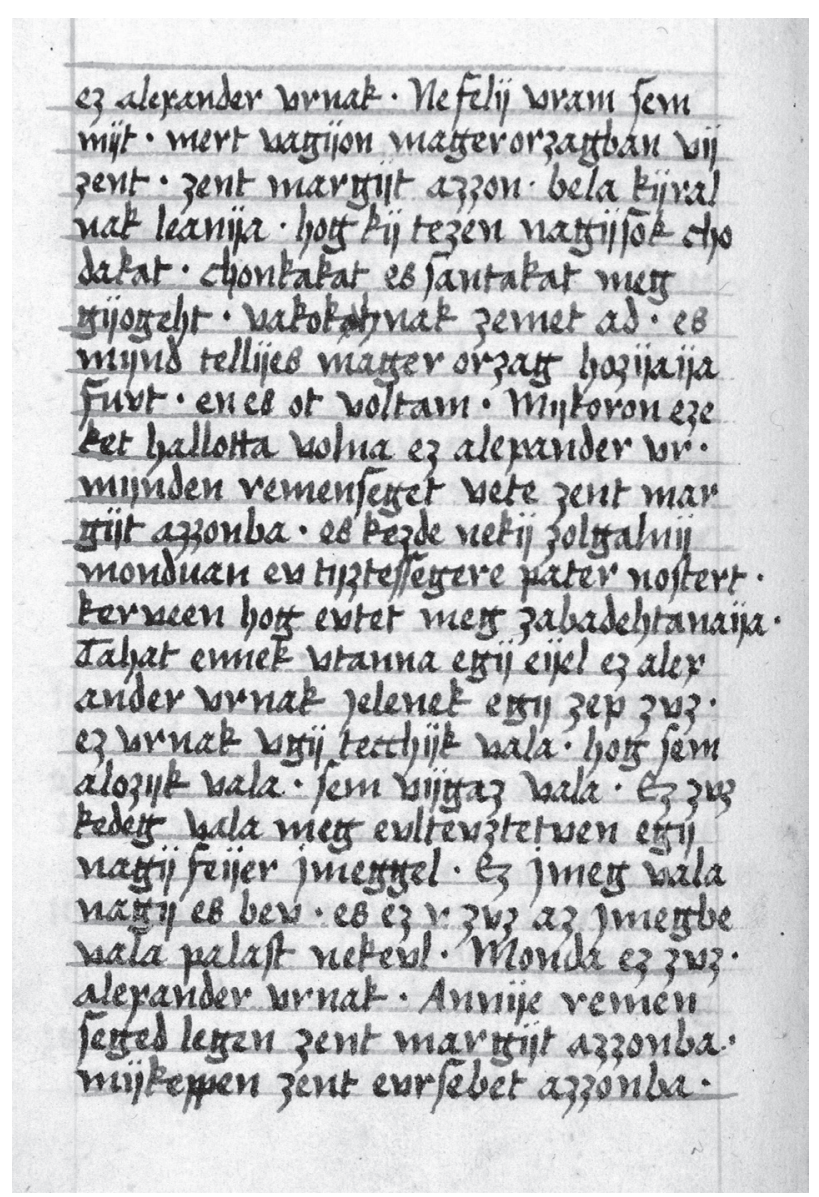

Fig. 1. Écriture de Lea Ráskay, BNH, MNY 3, f. 93v

Ráskay pourvut consciemment ses consœurs de lectures. Son écriture bâtarde ne devait pas causer de difficultés de lecture (fig. 1). De sa main, il nous reste aussi une lettre de la prieure Elena - également issue d'une famille de haute noblesse - adressée à son frère. De là, on peut déduire qu'elle occupait aussi des fonctions de secrétaire aux côtés de la prieure ${ }^{21}$. L'autre copiste dont nous connaissons le nom, Márta Sövényházi, nous a laissé son écriture dans deux codices. Elle orna elle-même le codex d'Érsekújvár, les préfigurations des miniatures de la série de la Passion étant des gravures sur bois

20. Lea Haader, «Arcképtöredékek ómagyar scriptorokról » [Portraits fragmentés de scripteurs de la Hongrie du Moyen Âge], in Látjátok feleim..., p. 53-74.

21. Lea Haader, «Elena priorissza levele » [La lettre de la prieure Elena], Magyar Nyelv, 91 (1995) p. 420-431. 
allemandes ${ }^{22}$. Dans l'ombre de la menace turque, Márta Sövényházi composa en 1529-1531 ce livre de grand format, particulièrement volumineux, qui proposait en soi une très grande variété de lectures pour toute l'année. Peut-être les sœurs s'apprêtaient-elles à fuir et le but était-il de créer un volume qui pût être utilisé dans n'importe quelle circonstance.

En $1541^{23}$, les sœurs se mirent effectivement en route, et à l'instar des clarisses de Óbuda elles fuirent devant les Turcs en direction du nord de la Hongrie. Les clarisses de Nagyszombat (aujourd'hui Trnava, Slovaquie) et de Presbourg (aujourd'hui Bratislava, Slovaquie) leur accordèrent l'asile. Dans leur fuite, elles avaient emporté leurs manuscrits et les reliques de sainte Marguerite. À leur extinction, les clarisses continuèrent à utiliser les codices du Moyen Âge, et ce jusqu'au XVII ${ }^{\mathrm{e}}$ siècle, comme l'attestent les annotations qui s'y trouvent. Malgré leur nombre limité, les manuscrits en langue hongroise provenant de ces couvents féminins sont proportionnellement beaucoup plus nombreux que les manuscrits latins des couvents d'hommes ou des chapitres. La raison en est que dans leur fuite ces derniers emportèrent leurs livres imprimés et naturellement aussi leurs chartes plutôt que les codices, déjà moins utilisés. Ceux-ci - dans l'intérieur du pays - ont été pour une large part détruits pendant les cent cinquante ans de la domination turque.

22. Tünde Wehli, «Az Érsekújvári Kódex illusztrációi » [Les illustrations du codex d’Érsekújvár)], in Látjátok feleim..., p. $159-170$.

23. Buda fut pris par les Turcs le 29 août 1541. 